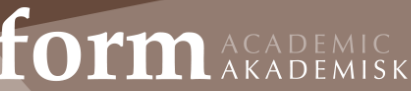

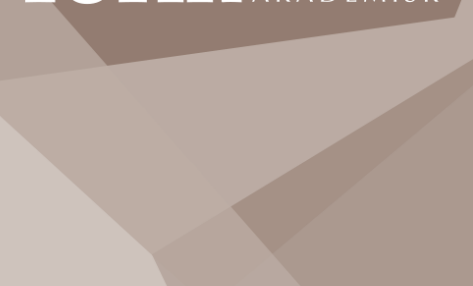

Vol I4, No I (2O2I)

\title{
Interactive architecture as a therapeutic environment for people with Alzheimer's disease, a scoping review
}

\begin{abstract}
As the global population ages, the number of people suffering from Alzheimer's disease (AD) increases. $A D$ is the most common cause of dementia. In recent years interactive architecture has been developed to enhance the lives of people coping with this disease. This article presents an extensive literature review from existent research projects on how assistive technology (AT) has been used as a physical and cognitive rehabilitation aid to $A D$ and other dementia patients. The review served to identify gaps in AT implemented place. That revealed the following findings: (1) a notable improvement in both physical and cognitive rehabilitation when integrating AT in patients' therapeutic environments, (2) a positive effect for caregivers when patients used AT individually, and (3) a lack of clarity due to limited studies on the use of AT for daily activities in residents' rooms at healthcare centers. However, further studies are necessary to explore the AT potential integrating strategies to promote daily activities in the residents' rooms at healthcare centers, and the architectural factors that could affect ATs' efficiency.
\end{abstract}

Keywords:

Assistive technology, Alzheimer's disease, interactive architecture, therapeutic environments, virtual environment.

\section{OVERVIEW}

This review is part of a cumulative doctoral dissertation comprising three substantial articles and an exegesis. The dissertation is on an interdisciplinary topic consisting of architecture, human-computer interaction $(\mathrm{HCl})$, and environmental psychology. Developing this research through interdisciplinary studies creates a powerful learning experience that emphasizes integrative learning, critical thinking, and creative problem solving. The aim of this dissertation is to develop the implementation of interactive indoor environments for persons with Alzheimer disease (PWAD) to support their orientation abilities in performing their daily tasks in their rooms at long-term healthcare centers in order to 
increase their quality of life (QoL). This aim leads to several related research questions: To what extent can interactive architecture add to the experience of an indoor therapeutic environment for people with Alzheimer's at long-term healthcare centers? What are the key system characteristics of integrating assistive technology (AT) as an assistive tool in residents' rooms at these centers? What kind of contributions could the use of virtual environments (VEs) in their rooms offer to PWAD? What are the architectural factors that could affect VEs' efficiency? What are the barriers to adopting VEs in residents' rooms? Which VEs could be used to motivate and orient the residents to do specific daily activity in their rooms (e.g., for toileting)? How can VEs be integrated in both single and multiple occupancy rooms? How do PWADs react to VEs? Studying residents' orientation in their rooms, how do they act before and after the use of VEs? What is the effect of the use of VE?

To answer these research questions, this dissertation targets objectives at three levels. The first objective is a scoping review to systematically map the research done in this area, as well as to identify any existing gaps in knowledge. The second objective is to study different assistive technology systems scenarios (which are used to motivate and orient residents' in their rooms to do a specific daily activity) through the user experience design concept, to investigate various aspects of using interior elements as information displays, taking into consideration the residents' as an end user. The third objective is to create a framework that can guide the researchers and designers to use interactive architecture in longterm healthcare centers for people with Alzheimer's.

\section{INTRODUCTION}

Alzheimer's disease (AD) is the most common type of dementia and account for between $50 \%$ and $75 \%$ of all cases (Alzheimer's disease and Dementia 2017). Cognitive scientists have defined AD as a progressive, chronic disease that severely impairs cognitive functions, including memory, reasoning, linguistic ability, depth perception, and mobility (Alzheimer's association 2018; National Institutes of Health, National Institute on Aging 2017; Alzheimer's disease International, n.d.). Van Hoof, Demiris, and Wouters (2017) provided an accurate definition of AD that gave a narrower perspective: precisely, it is the loss of necessary knowledge about "how to," including how to carry out the various tasks of daily living and how to interact appropriately with the environment. This definition provides an initial approach to resolving the larger problem at hand here. Therefore, prior understanding of the transition between disease stages is necessary (Mayo Clinic Staff). This transition may take several years, and patients in different stages of the disease have different requirements. Table 1 summarizes the familiar symptoms of $A D$ over its three stages.

TABLE 1: Alzheimer's Symptoms (Alzheimer's Society 2018b; Mayo Clinic Staff; Heerema, 2015)

\begin{tabular}{|c|c|c|}
\hline Stage & Activities Affected by Alzheimer's disease & Alzheimer's disease Symptoms \\
\hline $\begin{array}{l}\text { I. } \\
\text { Preclinical }\end{array}$ & $\begin{array}{l}\text { Memory, speech, complex organization, } \\
\text { social skills, judgment and logical thinking, } \\
\text { mobility, senses. }\end{array}$ & $\begin{array}{l}\text { A person may seem to be healthy but has increasing difficulty } \\
\text { making sense of the world around them. Short-term memory is } \\
\text { impaired, all skills and senses worsen, but substitutes may be } \\
\text { used to eliminate problems. }\end{array}$ \\
\hline $\begin{array}{l}\text { II. } \\
\text { Moderate }\end{array}$ & $\begin{array}{l}\text { Memory, speech, complex organization, } \\
\text { social skills, judgment and logical thinking, } \\
\text { mobility, senses. }\end{array}$ & $\begin{array}{l}\text { More intensive supervision and care become necessary, which } \\
\text { can be difficult for many families. The ability to take care of } \\
\text { oneself is lost, as is independent judgment. Orientation } \\
\text { deteriorates. }\end{array}$ \\
\hline III. Severe & $\begin{array}{l}\text { Cognitive ability to achieve anything } \\
\text { complex (including dressing oneself), } \\
\text { memory, mobility (often bed-bound). }\end{array}$ & $\begin{array}{l}\text { People in this stage of } \mathrm{AD} \text { cannot communicate and are } \\
\text { completely dependent on others for their care. They lose the } \\
\text { ability to walk, sit, and swallow and have increasing difficulty } \\
\text { communicating. }\end{array}$ \\
\hline
\end{tabular}


These cognitive problems have attracted substantial attention to the design and development of special care units for people with Alzheimer disease (PWAD). These units must provide a supportive environment for daily activities and increase PWAD's physical and mental independence. The loss of self-esteem linked with dependency in daily activities can be a devastating experience for PWAD (Yates et al., 2019).

PWAD need support to engage in activities that provide multisensory stimulation, as they may be incapable of accessing this type of stimulation by themselves. An adequate level of sensory stimulation helps to relieve stress and boredom. All the senses - sight, touch, taste, smell, sound, and movement-need stimulation. The senses related to movement can be divided into proprioception (the sense of where the body is in space) and vestibular awareness (awareness of velocity and direction of movement (Fowler, 2008).

Dementia-friendly design integrates the key principles that support maximum independent functioning without inducing anxiety (Adi et al., 2015). In incorporating these principles, architectural design aims to improve PWAD's vision and recognition by removing distracting objects, directing their attention to environmental cues, and highlighting key features in the surrounding environment. Virtual environment (VE) technologies can assist in this endeavour.

VE technologies are now a viable alternative to conventional rehabilitation methods. They have diverse properties and capabilities in patients' physical and cognitive rehabilitation-from conventional 2D graphic displays that provide no immersive or semi-immersive virtual reality (VR) scenarios to more advanced approaches, such as head-mounted displays and 3D smart TV technologies, with realistic multisensory interaction devices and neurophysiological feedback capacity. Such approaches are considered to be the most promising developments, as this review highlights. Additionally, it is desirable that these VR applications for PWAD be easily and affordably transferable to in-home and nursing home environments (García-Betances et al., 2015).

\section{METHODS}

The goal of the literature review is to inform primary research by (1) clarifying the importance of implementing a therapeutic environment for persons with Alzheimer's disease (PWAD) and identifying the characteristics of a positive outcome for these residents; (2) identifying the latest advances in technological solutions for interactive therapeutic environments in long-term healthcare centers for improving the PWAD's quality of life. The focus is on virtual environments (VEs) as assistive technology (AT) tools, (3) identifying the gaps in the AT-implemented place, looking at which types of AT have been integrated into the residents' rooms, the purpose of their usage, and whether the residents are using them individually or with caregivers' help. The literature review represents an embedded study, by collecting and analysing both quantitative and qualitative information. It involves a mixed research synthesis, by using quantitative research approaches to synthesize quantitative-based works and qualitative research approaches to synthesize qualitative-based works. The review is organized into two parts: (1) searching the literature and selecting relevant work and (2) categorizing the selected works and their use. In the first part; a systematic literature search was conducted in six scientific databases to identify relevant empirical studies. A focus was on the databases related to health, nursing, environmental psychology, the built environment, $\mathrm{HCl}$ and assistive technology. Therefore, a literature search was performed in PubMed, Web of Knowledge, IEEExplore, ScienceDirect, and Google Scholar electronic databases, using the following keywords: assistive technology, interactive architecture, therapeutic environment, virtual environment, multisensory stimulation, multisensory environment, and augmented reality. The keywords were combined with the terms "dementia" and "Alzheimer's" to identify papers on the topic, as well as pilot studies. Published studies were next identified using a search strategy based on the three facets of the research question: persons with Alzheimer's disease and other dementia, interactive therapeutic environments, and long-term healthcare centers. Only Englishlanguage peer-reviewed journal articles published after 2003 were considered in investigating the importance of the therapeutic environment. Articles published after 2014 were particularly considered to examine the use of more recent technologies. Eligible articles were those that examined the effects 
of no immersive, semi-immersive, and/or fully immersive virtual, augmented, or mixed reality interventions using head-mounted devices on participants' quality of life, depressive symptoms, social interaction, enjoyment, and acceptability. In the second stage of the review, the retrieved VR studies and applications were categorized according to the classification in Figure 1.

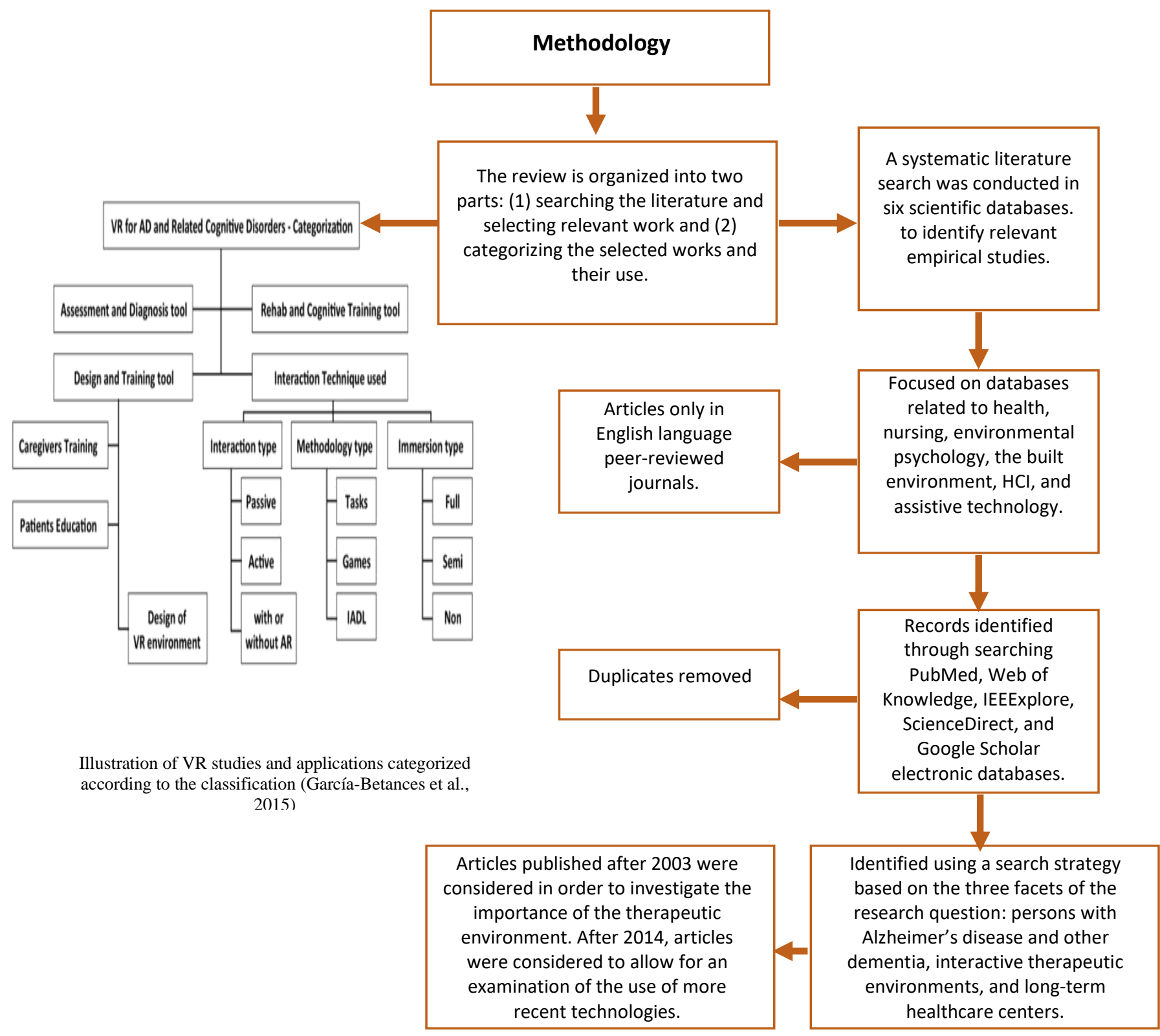

FIGURE 1. Flowchart of the literature search on the Interactive architecture as a therapeutic environment for people with Alzheimer disease. 


\section{RESULTS}

This section outlines the results of the literature review. Numerous studies have confirmed a positive correlation between the therapeutic environment design (especially when healthcare architects consider patients as end users) and patient improvement (Bowes \& Dawson, 2019; Zeisel, 2000, 2003; Malkin, 1992; Marquardt et al., 2014; Smith \& Watkins, 2016; van Hoof et al., 2017). Including interactive architecture in patients' environments improved their physical and cognitive rehabilitation and their self-esteem (Marquardt et al., 2014; McCullough, 2005; Yates et al., 2019). VEs play an important role in the improvement of PWAD's physical and cognitive rehabilitation, including in such areas as memory retention, attention, and problem solving (Dalton 2017; Fasilis et al. 2018; Hofmann et al. 2003; Moyle et al. 2018). However, there are some gaps concerning the integration of VEs in residents' rooms. Other limitations include a lack of studies on the effects of architectural factors on VEs' efficiency, including illumination (day and artificial light), materials (ordinary and smart materials), and the room area (James R. Benya, 2010). In addition, the role of VE integration in rooms with multiple residents in healthcare centers remains unclear (Dalton, 2014).

\section{THERAPEUTIC ENVIRONMENT ROLE}

Recently multiple studies ( Marquardt et al., 2014; Smith \& Watkins, 2016; Zeisel, 2000, 2003) have acknowledged the relation between therapeutic environments and patient improvement. Many researchers highly recommend considering the patient as an end user in the design process, as this contributes to a more supportive therapeutic environment (McCullough, 2005). The research literature in this field has grown rapidly in recent years. For instance, Bowes and Dawson (2009) have systematically identified, examined, and evaluated studies on designing environments for people with dementia (PwD). Marquardt et al., (2014) indicated that specific design interventions improve outcomes for PwD, except in the area of their cognition.

Zeisel, (2003) examined indications of the most appropriate treatments for PWAD in order to better understand the therapeutic environment (environment, behavior/communication, and medication). Other research has focused on the use of technology in the therapeutic environment for PWAD and other types of dementia. One study (Topo, 2009) suggested that universal design principles can be a helpful starting point, but these must be supplemented with dementia-specific knowledge. This requires that PWD be involved in the design process and implies that it is essential for technologies to be thoroughly tested in a real-world environment and not solely in laboratory conditions. Another study (Dalton, 2017) described a framework for a smart home environment that aims to comprehensively address issues of environmental fit, in particular for people with dementia. This includes a means of sensing the user affect as a factor in the system management of a smart personal living space and generating environmental responses that adapt to changing user needs. The overall intention is to maximize environmental congruence for the user, both functionally and psychosocially, by factoring in adjustments based on changing patient status.

\section{INTERACTIVE TECHNOLOGIES}

Using interactive technology in everyday life can help PWAD maintain their independence, keep safe, and stay active and involved (Alzheimer's Society, 2019). This technology can assist individuals in the process of remembering, including the receiving, encoding, storing, and retrieving of memories. Memory problems can be caused by difficulties at any of these stages. A useful way to improve the process of remembering is to establish a daily routine and break it into smaller steps (Alzheimer's Society, 2018b). Assistive technology (AT) can play an important role in this process. AT refers to devices or systems that help maintain and improve a person's ability to function in everyday life. Such technologies can assist with many difficulties, including problems with memory and mobility. AT includes a broad range of items, ranging from electronic pill boxes to "smart home" systems 
(Alzheimer's Society, 2018a). This section focuses on the main developments in AT and locating evidence as to whether AT can improve the well-being of PWAD.

\subsection{Interactive technology effectiveness and issues}

Recent literature reviews (Clay et al., 2020; D'Cunha et al., 2019; García-Betances et al., 2015; Koumakis et al., 2019; Adi \& Aljunaidy, 2020; Montana et al., 2019; Sánchez et al., 2013; Strong, 2020) and position papers have addressed AT for PWD and PWAD from a broad perspective. Generally, these publications discuss a range of topics to advance research and bring to market evidence-based solutions that are usable and effective in patients' everyday lives. While AT has potential benefits to offer PWD (Alzheimer's Society, 2018a), there are still a number of general issues around its provision that need to be explored in detail. These include improving access, personalized care, analysis of limitations, ethical considerations, and future developments.

A conference paper confirmed the lack of standards for relating quality of life (QoL) to AT (Hayhurst, 2017). A number of ATs, including virtual reality (VR) and augmented reality (AR), have been reported to enhance the QoL of PWAD. However, It can be difficult for PWAD and care personnel to evaluate the products available to them (Hayhurst, 2017). Therefore, in future research, technologybased QoL metrics would support the objective assessment of the impact of AR and VR.

"Interaction design must serve the basic human need for getting into place. Like architecture, and increasingly as a part of architecture, interaction design affects how each of us inhabits the physical world" (Alzheimer's association, 2019). Experts have stated that while some technology tools can be helpful for PWAD, others do not work as promised. James Hendrix, director of global science initiatives at the Alzheimer's Association, stated that "there exist hundreds of products and computer apps to help PWAD, but very few are clinically proven" (Alzheimer's Society, 2019).

Several studies that examined the use of AT for PWAD have shown promising results. For example, a case study of night-time wandering (Kenfack Ngankam et al., 2020) showed the importance of context awareness architecture for ambient assisted-living applications. Another study presented and reflected on recent findings on how PWD experience and manage their daily lives through their own initiative, including their use of technology (Topo, 2009). Yet another study evaluated a novel kind of interactive computer-based cognitive training in $A D$ for rehabilitation and therapeutic interventions (Hofmann et al., 2003).

\subsection{Virtual environments as cognitive aids for people with Alzheimer's}

VEs are a promising technology that can be used for cognitive assessment and intervention (Alzheimer's association, 2019). VR immerses the user in a dynamic VE in which they carry out cognitive and sensorimotor activities while interacting with virtual stimuli. The use of interaction in VEs provides new approaches to the treatment of memory deficits in elderly individuals (Corriveau Lecavalier et al., 2020). Recent literature (Clay et al., 2020; D'Cunha et al., 2019; Sánchez et al., 2013; Strong, 2020) has shown the effectiveness of VEs in improving patients' performance on several tasks in physical and cognitive rehabilitation, using immersive, semi-immersive, and no immersive options. These tasks include reminiscence activities, such as using an interactive touchscreen device to involve discussing events and experiences from the past and aims to evoke memories, stimulate mental activity and improve a person's well-being. As well as spatial cognitive tasks, and enjoyable leisurely activities.

In addition, several pilot studies have shown promising results in the use of VR technologies as cognitive aids for PWAD. One study (Fasilis et al., 2018) examined the effect of computerized cognitive rehabilitation and interactive computer-based training on potential cognitive enhancement and rehabilitation in persons with mild dementia. This study found a relative improvement in patients' cognitive functions, including those related to problem solving, rigid thinking, and attention. Another pilot study (Optale et al., 2010) implemented a VR training intervention to try to reduce cognitive decline and improve memory functions. Moyle et al., (2018) measured and described the effectiveness of a VR forest on engagement, apathy, and mood states of PWD and explored the experiences of staff, PWD, and 
their families. A study by Manera et al., (2016) tested the feasibility of using highly realistic image-based rendered VR with people with mild cognitive impairment and dementia. The researchers designed an attentional task to train selective and sustained attention and tested VR and paper versions of this task in a single session. The above studies demonstrate that VR-based training can be a tool for improving adherence to cognitive training among PWAD.

Using both VR and AR, Hayhurst, (2017) addressed several technology design issues with VEs, the first being the consideration of the stakeholders. AT often involves a level of technical intervention, either to set up the application or to use it, which requires that caregivers or family members be able to use such applications to support PWD. Furthermore, the designers of AT need to follow a usercentered approach rather than a one-size-fits-all approach. Any intervention using AR or VR must be designed considering the specific needs of the individual with dementia, as their needs may differ depending on how their dementia is affecting them. AR and VR could be developed to support VEs that are context-aware of PwD, thus resulting in a more immersive adoption of any intervention.

\section{VIRTUAL ENVIRONMENTS AND ALZHEIMER'S-FRIENDLY BUILDING DESIGN}

VEs stimulations can be used in various scenarios for health facility design. These include immersive, semi-immersive, and no immersive scenarios. Most research related to VEs and AD has focussed on enhancing the specific skills that tend to decline over the course of the condition, including spatial navigation (Jiang \& Li, 2007; Montana et al., 2019; Pengas et al., 2012; White \& Moussavi, 2016). The results are promising and suggest that VR training can facilitate neuro-rehabilitation and promote brain plasticity ${ }^{1}$ processes. Applications of virtual buildings and rooms can both evaluate and rehabilitate the spatial memory of patients in different cognitive impairment phases.

Other studies have focused on creating multisensory spaces to enhance cognitive skills (Duchi et al., 2019; Goodall et al., 2019). This stimulation of sight, touch, hearing, balance, and smell is expected to lead to a reconnection with reality for PwD, resulting in an improvement in their overall well-being and QoL. In addition, it can help patients improve their relationships in their social and personal environments, since the aim is to provide an atmosphere of wellness and relaxation for the patient and the specialist.

Eisapour et al., (2018) created two VR environments using the Oculus Rift head-mounted display and Oculus touch controllers with the goal of increasing accessibility to exercise for PwD. This research demonstrates the promising potential of VR exergames ${ }^{2}$ for PWD. However, future studies are needed to expand the available tasks, increase the available environments, and examine their clinical impact. Suggestions have been made for dementia-friendly room designs, but the effectiveness of these in improving the safety of dementia patients has not yet been tested (Dalton, 2014, 2017). The author noted that the ideal environment for PwD should be adaptable to compensate for the loss of cognitive function and to decrease the patient's susceptibility to stress of environmental origin, so that the room environment becomes prosthetic or compensatory. This research used Barris's model of environment, including the following aspects: (1) objects; (2) tasks, meaning any series of actions that satisfy internal motives to explore and be competent; (3) groups, since the care setting architecture can either advance or limit connections to the greater physical and social context; and (4) culture, referring to family culture, as the design context is a conscious recognition of the user's or resident's expectations. (Dalton, 2014,2017 ) suggests that congruence in the architectural environment of care can be supported by the incorporation of real-time sensing and actuation. In such an environment, affect becomes a consideration in the management of environmental response, thus creating a feedback loop that promotes salutogenesis3.

\section{DISCUSSION}

$A D$ is a progressive disease, the symptoms of which differ from person to person (Alzheimer's association, 2018; Alzheimer's Disease International, n.d.; National Institutes of Health, National Institute on Aging, 2017). In all cases (Alzheimer's Society, 2018b), the problem is on of "how to," as AD 
and other kinds of dementia often make performing the activities of daily living difficult. Tasks may be done halfway, poorly, or not at all (Heerema, 2015). Therefore, healthcare designer should focus on the remembering process when designing AD-patient-friendly buildings. The improvement of patient outcomes depends on the therapeutic environment, as several pilot studies have shown theoretically and practically.

As Smith \& Watkins (2016) mentioned, the effects of environments can be either positive or negative; only no environment is neutral. One of the first authors to describe the therapeutic environment effect (Malkin, 1992) listed the following recommendations: (1) reduce or eliminate environmental stressors, (2) provide positive distractions, (3) enable social support, and (4) give patients a sense of control. These factors all influence the effect of the therapeutic space on patient outcomes. In addition, Zeisel $(2000,2003)$ concluded that for positive therapeutic environment outcomes, specifically for PWAD, healthcare architects should first classify the relevant symptoms and their treatment. A useful classification scheme of Alzheimer's symptoms includes the behavioral, functional, cognitive, and physical manifestations. By identifying AD's relevant symptoms, architects can design the space to help reduce one or more of them.

Zeisel's $(2000,2003)$ recommendation leads to another aspect of the space: the service to the PWAD who could be served by the space itself. Considering the PWAD as an end user will lead to a positive outcome (McCullough, 2005). Bowes \& Dawson (2019) provided a foundation for the further development of practical design work on environments that enable people with dementia to live better. They concluded that there is currently disparate work available on a range of design items, prototypical technologies, and interventions, and that research is needed to consolidate findings and identify the core principles to guide design. In addition, they considered the environment not only as facilitating and supporting good care but also as having a positive role to play in itself.

Dalton (2017) and Topo (2009) proved that patients had better outcomes when AT was integrated into their therapeutic environments, which increased their autonomy and independence. AT can be used in daily activities that exercise the remembering processes (Alzheimer's association, 2019; Alzheimer's Society, 2018b, 2019), and a daily routine divided into small steps can improve patient outcomes. As previously discussed, there are still a number of general issues around the provision of AT that need to be explored and addressed in greater detail (Alzheimer's Society, 2018a). These include the following:

1. Improved access. This issue encompasses two points: (1) A lack of public awareness and information, means that PWAD and their carers do not know what to ask for. (2) A lack of professional awareness, means that there is also a need for Alzheimer's advisers and other memory services staff to receive AT awareness training.

2. Personalized care. It is very important that AT is personalized to the individual and not part of a 'set menu' or 'Alzheimer's package'. PWAD experience various symptoms that require different responses. In addition, the most appropriate AT will depend on an individual's lifestyle and circumstances, which change over time. This confirms the theory that 'no one design can fit all'.

3. Limitations. AT should not be treated as an 'amend solution' for PWAD or used as a replacement for human interaction and caring for them. Rather, AT should be seen as complementing an individual's care and support to enhance their QoL. The AT design process should include PWAD to produce technological aids that are 'fit for purpose' without being overly complex or requiring extensive training. The key to some of the issues around AT for people with $A D$ and caregivers is how reliable off-the-shelf technical solutions are. For example, for some PWAD telecare is of use only if it is backed up with human care, support, and training. How useful AT is also depends strongly on the external environmental factors that are in place. For example, adequate lighting can be a significant factor in determining whether PWAD are able to navigate around their home or environment. 
4. Ethical considerations. To fully realize the beneficial effects of the technology, particular attention should be paid to aspects of care planning including assessment, installation, and obtaining consent.

5. Future developments. The proper promotion of AT benefits requires strong local and national leadership, with advocates explaining the ways in which AT can bring benefits to PWAD and their careers. AT can play an important role in several daily life activities of PWAD (Hofmann et al., 2003; Kenfack Ngankam et al., 2020; Topo, 2009), as it has been shown training improved the task performance of PWAD substantially, and they appeared to like this approach. New interactive media, therefore, may yield interesting opportunities for rehabilitation and therapeutic interventions (Hofmann et al., 2003).

One promising form of AT is VEs, which are considered effective rehabilitation tools for PWAD (Alzheimer's association, 2019; Corriveau Lecavalier et al., 2020). As previously mentioned, recent literature (Clay et al., 2020; D'Cunha et al., 2019; Sánchez et al., 2013; Strong, 2020) has proved the effectiveness of VEs in improving patients' performance on several tasks through the use of immersive, semi-immersive, and no immersive scenarios. These tasks include reminiscence activities and spatial cognitive tasks that involve enjoyable, leisurely activities. Some people have argued that PWAD will not enjoy or interact with VEs, but research has shown that PWAD have indeed enjoyed and interacted well with VEs. Additionally, there was some improvement in their QoL and memory function after using VEs in some tasks such as path finding, problem solving, and tasks to stimulate attention (Fasilis et al., 2018; Hayhurst, 2017; Manera et al., 2016; Moyle et al., 2018; Optale et al., 2010).

Although the use of VEs in patients' physical and cognitive rehabilitation has improved outcomes, there are still some problems that need to be solved. For instance, the set-up and use of some devices, such as headsets, tablets, and screens, requires a level of technical intervention. This requires that caregivers have the ability to use such applications to support PWAD in their use of them. A further problem is that caregiver intervention of this type will not help PWAD become more selfsufficient. Moreover, not all VE designs fit all patients. Instead, designs are required that consider PWAD as end users with individually specific needs (McCullough, 2005).

The use of VEs for AD has developed recently in the architecture field. VEs have been used in various ways in architectural design to improve AD patient outcomes, and several studies have shown their benefits for these patients. The four main factors in the use of VEs are as follows: (1) the PWAD as an end user, (2) the space the VE will be integrated into, (3) the type of VE, and (4) the assistive service that the technology will provide to the PWAD. Table 2 illustrates this categorization. 


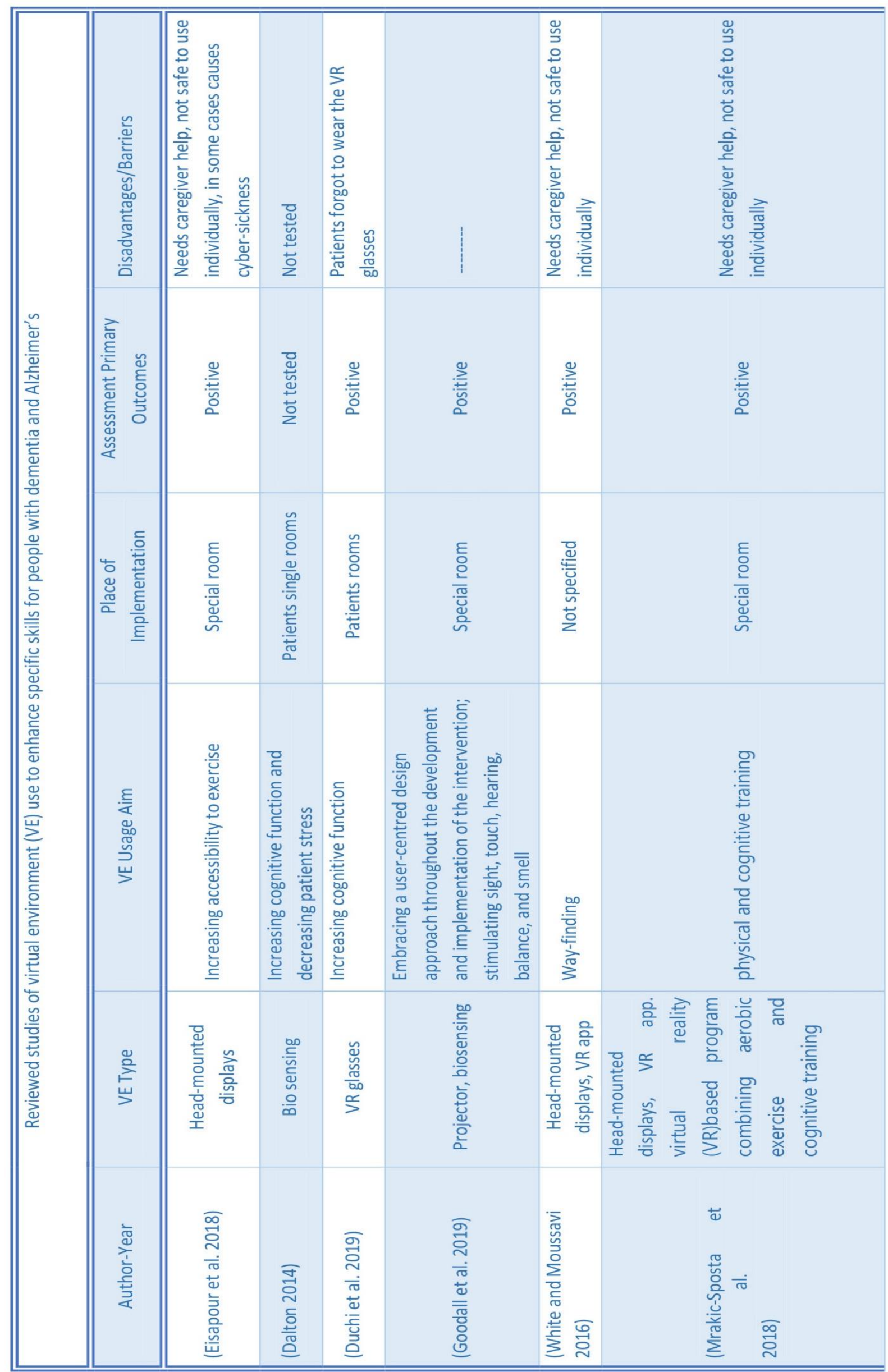

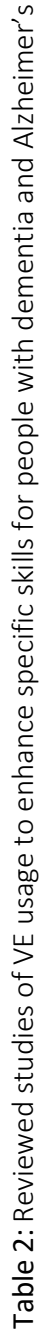


The table can be summarized as follows. (Eisapour et al., 2018) investigated physical rehabilitation for PWD using VR exergames in a special room under caregivers' supervision. The duration of this rehabilitation was three weeks, and was provided to six persons living with dementia. The researchers created two VEs using an Oculus Rift head-mounted display and Oculus touch controllers. An evaluation compared the virtual programs with human/therapist-guided exercise in terms of the subjective enjoyment, comfort, and difficulty level of the activities. The evaluation showed positive outcomes in the PWAD's physical rehabilitation that exceeded those gained from ordinary guided exercise.

Another study (Dalton, 2014) suggested using VEs to enhance PWAD's QoL in addition to assisting with cognitive rehabilitation by integrating biosensors in the residences' single-occupancy rooms at Alzheimer's healthcare centers. The author used Barris's model of the environment, looking at the aspects objects, tasks, groups, and culture. The 'My Room' concept envisioned patient rooms designed to include a comprehensive embedded responsive system, with multiple networked sensors, allowing continuous activity-aware and contextualized sensing of a user's psycho-physiological state. This suggestion has not been tested in real life. The key characteristics of the My Room model are summarized in Figure 2.

\begin{tabular}{|c|}
\hline Functional Congruence Pser-Centred \\
Architecture as Interactive Design \\
Person Context \\
\hline Environmentally Embedded Sensing \\
\hline Sensing of Person \\
Real-Time Environmental Response \\
\hline $\begin{array}{c}\text { Distributed System } \\
\text { Intelligence } \\
\text { Feedback Loop/Open Work }\end{array}$ \\
\hline
\end{tabular}

FIGURE 2. System characteristics of an adaptive salutogenic room (Dalton 2014)

Goodall et al. (2019) examined physical and cognitive rehabilitation in addition to QoL for PwD by using an immersive VE that combined VR and AR in a special room that was automatically adaptable to the personal memories and individual preferences of the users. The design of the space is shown in [Figure 3]. The researcher combined multisensory stimulation with physical activity and techniques from reminiscence therapy and Montessori methods. This stimulation of sight, touch, hearing, balance, and smell was expected to lead to a reconnection with reality for the PWD. The researcher used a projector to display information onto a large wall. The VE combined an AR game to improve the patient's balance and physical activity and integrated an interactive touchscreen device showing family photographs from the individual's life story to improve the patient's sense of touch. In addition, an integrated sound system with familiar music and background soundscapes was used to motivate and enhance the patient's cognitive rehabilitation. To complete the scene for the patients, an olfactory dispensary system released familiar scents.

This case study demonstrated the effectiveness of the VE use in two areas. First, it had a positive outcome on patients' physical and cognitive rehabilitation and improved their QoL. Second, it had a positive effect on both the formal and informal caregivers. The improvement in caregiver coping and relief from stress, in turn, increased the quality of informal caregivers' visits to PWD and enhanced the quality of their relationships. In this study, patients used the VE without caregivers' help, and it was safe to be used individually. 


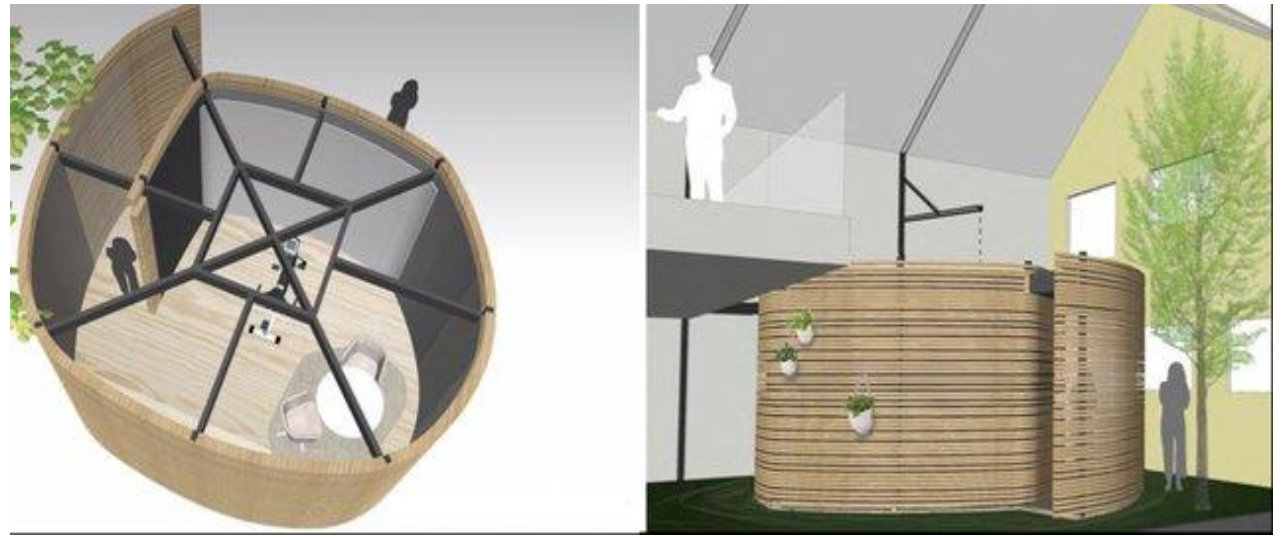

FIGURE 3. Architectural sketches of the SENSE-GARDEN space (left: interior, right: exterior Goodall et al. 2019)

Other studies by (Mrakic-Sposta et al., 2018; White \& Moussavi, 2016) that investigated patients' cognitive rehabilitation, specifically for spatial cognitive tasks, suggested that PWAD are able to transfer information about the environment obtained from the VE to real life. The study used a cognitive treatment program based on spatial navigation in a VE, having a man with early-stage AD navigate to targets in a symmetric, landmark-less virtual building. The treatment took place in a special room under a caregiver's supervision. The study proved that an individual in the early stages of AD could learn to navigate in a simple VR navigation environment, suggesting that such treatments might benefit other people with AD.

The use of head-mounted displays and computer applications, however, comes with disadvantages. In particular, it will not increase patients' self-esteem, as it must be done under caregiver supervision and in specific spaces. In addition, some studies have confirmed that head-mounted displays can cause cyber-sickness and anxiety for seniors with $A D$ or other forms of dementia (Nesbitt \& Nalivaiko, 2020; Pot-Kolder et al., 2018).

By analyzing the previous table through the four main aspects of the positive therapeutic environment outcomes which are; (1) reduce or eliminate environmental stressors, (2) provide positive distractions, (3) enable social support, and (4) give PWAD a sense of control. Had been found that the use of fully immersive systems will not develop a therapeutic environment with a positive outcome. As there's a lack on aspects one and four. In this sense, display screens and semi-immersive systems, are more comfortable to use. Although, (Goodall et al. 2019) had all the aspects of the effective therapeutic environment, but the system had been integrated into a special room without any window. As the lack of sufficient exposure to bright light during the day can negatively affect the health and well-being of residents in Alzheimer's and other dementia healthcare centers (Hanford \& Figueiro, 2013; Konis et al., 2018; Torrington \& Tregenza, 2007)

This leads to the identification of the main research gap, which relates to the orientation of PWAD in their rooms by having them use VEs as assistive technology tools to guide them in performing specific daily activities (e.g., toileting, dressing, self-care, etc.), which differ from one patient to another. The next step of the dissertation will be divided into two parts: (1) observing the PWAD's orientation in their rooms at healthcare centers and (2) studying assistive technology as an assistive tool to motivate and orient them in performing specific daily activities through the design of experience concept and using a mixture of qualitative and quantitative methods, including (1) data collection through a survey and interviews by professionals at an Alzheimer's long-term healthcare center in Vienna, Austria, as well as through observing residents; (2) analysis of the collected data; and (3) developing a simulation for the residents' orientation in their rooms to create a narrative scenario for their orientation, and to discover the influence of architectural elements on the efficiency of the assistive systems. The survey will address the following questions: what are the residents' needs in their rooms? What are their daily activities during the day and night? What type of assistive technology are they using? What are the reasons for this usage? Where do they use each type and when? At which stage of AD do they use them? How do they interact with the assistive technology? What are the architectural barriers while using the 
assistive technology in their rooms? From a survey completed by professionals ( $n=25)$ and interviews held with specialists $(n=5)$ in an Alzheimer's long-term healthcare center in Vienna, Austria, the following daily activities were chosen to be investigated in the current study: (1) orient to bed, (2) orient to toilet [Figure 4], (3) orient to wardrobe, and (4) orient to dining table.

\section{room}

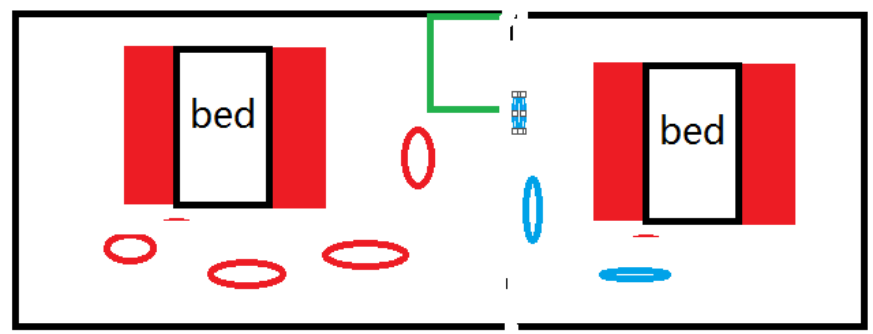

FIGURE 4. illustrate sketch of the residents' movement to the toilet door in one of the doubled room at the healthcare canter, Vienna, Austria.

\section{CONCLUSION}

This literature review of 55 articles, with a focus on interactive architecture in the therapeutic environment for PWAD, confirmed that including interactive architecture in residents' environments enhanced their rehabilitation and self-esteem (Clay et al., 2020; D'Cunha et al., 2019; Sánchez et al., 2013; Strong, 2020). The main problem for PWAD is the process of remembering "how to." Every patient experiences a different situation, however, which confirms the theory that "no one design fits all." Therefore, healthcare architects and interior designers need to consider PWAD as end users. In general, the reviewed articles highlighted the importance of the therapeutic environment and its relation to outcomes in PWAD. They identified the main design aspects that should be considered by healthcare designers, as follows: (1) reducing or eliminating environmental stressors, (2) providing positive distractions, (3) enabling social support, and (4) giving a sense of control. In addition, the versatile uses and positive effects of adopting ATs in the therapeutic environment were also apparent, including improving QoL, facilitating rehabilitation, and enhancing socialization. The studies confirmed that PWAD are able to interact with ATs and that they enjoy using them.

Immersive, semi-immersive, and no immersive VEs have a positive impact on PWAD outcomes, but some issues with VEs (using VR or AR) remain problematic. One issue is that most ATs require the intervention of caregivers, which, overall, does not help to increase patients' self-esteem. Additionally, some studies found that head-mounted displays can cause cyber-sickness and anxiety for some patients. The literature shows that PWAD have difficulty with way-finding, but that they can learn their way if the environment is supportive (Cushman et al., 2008; Taillade et al., 2013). VR applications for the early detection of dementia, including VR Practice, VR Park, and VR Games, have been developed to assess the spatial memory of early-stage dementia patients (Wen et al., 2018). Researchers have used VR and AR in the spatial domain by applying different devices for different purposes. Some have used headmounted displays with biosensors, while others have used VR glasses and interactive projectors with biosensors in dedicated rooms. Experiments have indicated that all these devices have some effectiveness, but this varies from one patient to another, depending on their condition.

There is a need for future studies to be designed with the aim of improving residents' orientation in their rooms by their using VEs individually as assistive tools to motivate and orient them to do a specific daily activities, which may differ from one resident to another. Moreover, there is a need for more research focusing on the implementation of interactive environments in multiple residence rooms at long-term healthcare centers, the use of VEs to stimulate patients in carrying out their daily activities, and the effect of architectural factors on the efficiency of VEs. 
ACKNOWLEDGEMENTS

The work described in this paper is part of a doctoral dissertation. 


\section{REFERENCES}

Adi, M. N. \& Aljunaidy, M. M. (2020). The usefulness of using virtual reality to assess elderly and dementia friendly hospital design. IDA: International Design and Art Journal, 2(1), 137-150. http://www.idajournal.com/index.php/ida/article/view/31

Adi, M. N., Parke, B. \& Friesen, K. (2015). Code plus: Physical design components for an elder friendly hospital, 2nd edition. Fraser Health Authority. https://www.researchgate.net/publication/280722860_Code_Plus_Physical_Design_Components_for_a n_Elder_Friendly_Hospital_2nd_Edition

Alzheimer's association (2018). 2018 alzheimer's disease facts and figures, 14(3), 367-429. https://doi.org/10.1016/j.jalz.2018.02.001

Alzheimer's association. (2019). Technology's Evolving and Expanding Role in Dementia Care, Prevention and Alleviating Burden [Press release]. Amsterdam, Netherlands. https://www.alz.org/aaic/releases_2019/sunTECHNOLOGY-jul14.asp

Alzheimer's Disease International. (n.d.). Alzheimer's disease. https://www.alz.co.uk/info/alzheimers-disease

Alzheimer's Society (2018a, March). Alzheimer's society's view on assistive technology. https://www.alzheimers.org.uk/about-us/policy-and-influencing/what-we-think/assistive-technology

Alzheimer's Society (2018b). The memory handbook: a practical guide to living with memory problems. Alzheimer's Society.UK, 64. https://doi.org/10.1159/000491488

Alzheimer's Society (2019, April). Using technology to help with everyday life. https://www.alzheimers.org.uk/sites/default/files/2019-05/437LP-Using-technology-to-help-witheveryday-life-190520.pdf

Benya, J. R. (2010, March 29|). Controlling glare: deciphering this technical condition to create responsive lighting solutions. Architect. https://www.archlighting.com/projects/controlling-glare_o

Bowes, A. M., \& Dawson, A. (2019). Designing environments for people with dementia: A systematic literature review. Emerald Publishing. https://doi.org/10.1108/9781787699717

Clay, F., Howett, D., FitzGerald, J., Fletcher, P., Chan, D., \& Price, A. (2020). Use of immersive virtual reality in the assessment and treatment of alzheimer's disease: A systematic review. Journal of Alzheimer's Disease : JAD. 75(1), 23-43. (Advance online publication). https://doi.org/10.3233/JAD-191218

Corriveau Lecavalier, N., Ouellet, É., Boller, B., \& Belleville, S. (2020). Use of immersive virtual reality to assess episodic memory: A validation study in older adults. Neuropsychological Rehabilitation, 30(3), 462-480. https://doi.org/10.1080/09602011.2018.1477684

Dalton, C. (2014). Myroom: A user-centred model of affective responsive architecture: myroom: A user-centred model of affective responsive architecture [PhD Thesis, University College Cork]. https://cora.ucc.ie/handle/10468/1860

Dalton, C. (2017). Including Smart Architecture in Environments for People with Dementia. In J. van Hoof, G. Demiris \& E. Wouters (Eds.), Handbook of Smart Homes, Health Care and Well-Being (Vol. 9). Springer Cham. https://doi.org/10.1007/978-3-319-01583-5_57

D'Cunha, N. M., Nguyen, D., Naumovski, N., McKune, A. J., Kellett, J., Georgousopoulou, E. N., Frost, J., \& Isbel, S. (2019). A mini-review of virtual reality-based interventions to promote well-being for people living with dementia and mild cognitive impairment. Gerontology, 65(4), 430-440.

https://doi.org/10.1159/000500040

Duchi, F., Benalcázar, E., Huerta, M., Bermeo, J. P., Lozada, F., \& Condo, S. (2019). Design of a multisensory room for elderly people with neurodegenerative diseases. In L. Lhotská, L. Sukupova, I. Lacković, \& G. S. Ibbott (Eds.), IFMBE Proceedings: World Congress on Medical Physics and Biomedical Engineering 2018: June 38, 2018, Prague, Czech Republic. (Vol. 68/3, pp. 207-210). Springer. https://doi.org/10.1007/978-98110-9023-3 37 
Eisapour, M., Cao, S., Domenicucci, L., \& Boger, J. (2018). Virtual reality exergames for people living with dementia based on exercise therapy best practices. Proceedings of the Human Factors and Ergonomics Society Annual Meeting, 62(1), 528-532. https://doi.org/10.1177/1541931218621120

Fasilis, T., Patrikelis, P., Siatouni, A., Alexoudi, A., Veretzioti, A., Zachou, L., \& Gatzonis, S.-S. (2018). A pilot study and brief overview of rehabilitation via virtual environment in patients suffering from dementia. Psychiatrike = Psychiatriki, 29(1), 42-51. https://doi.org/10.22365/jpsych.2018.291.42

Fowler, S. (2008). Multisensory rooms and environments: Controlled sensory experiences for people with profound and multiple disabilities (JKP resource materials). Jessica Kingsley Publishers.

García-Betances, R. I., Arredondo Waldmeyer, M. T., Fico, G., \& Cabrera-Umpiérrez, M. F. (2015). A succinct overview of virtual reality technology use in alzheimer's disease. Frontiers in Aging Neuroscience, 7, 80. https://doi.org/10.3389/fnagi.2015.00080

Goodall, G., Ciobanu, I., Taraldsen, K., Sørgaard, J., Marin, A., Draghici, R., Zamfir, M.-V., Berteanu, M., Maetzler, W., \& Serrano, J. A. (2019). The use of virtual and immersive technology in creating personalized multisensory spaces for people living with dementia (sense-garden): Protocol for a multisite before-after trial. JMIR Research Protocols, 8(9), e14096. https://doi.org/10.2196/14096

Hanford, N., \& Figueiro, M. (2013). Light therapy and alzheimer's disease and related dementia: Past, present, and future. Journal of Alzheimer's Disease : JAD, 33(4), 913-922. https://doi.org/10.3233/JAD-2012121645

Hayhurst, J. (2017, February). How augmented reality and virtual reality is being used to support people living with dementia: design challenges and future directions. In. T. Jung \& M. C. tom Dieck (Eds.), Augmented Reality and Virtual Reality - Empowering Human, Place and Business. Manchester Metropolitan University https://www.researchgate.net/publication/315053361_How_Augmented_Reality_and_Virtual_Reality_i s_being_used_to_support_people_living_with_Dementia_-_Design_challenges_and_future_directions

Heerema, E. (2015, March 30). Dementia effects on activities of daily living (adls). Verywell Health. https://www.verywellhealth.com/dementia-daily-living-adls-97635

Hofmann, M., Rösler, A., Schwarz, W., Müller-Spahn, F., Kräuchi, K., Hock, C., \& Seifritz, E. (2003). Interactive computer-training as a therapeutic tool in alzheimer's disease. Comprehensive Psychiatry, 44(3), $213-$ 219. https://doi.org/10.1016/S0010-440X(03)00006-3

Jiang, C.-F., \& Li, Y.-S. (2007). Virtual hospital--a computer-aided platform to evaluate the sense of direction. Conference Proceedings: Annual International Conference of the IEEE Engineering in Medicine and Biology Society. 2007, 2361-2364. https://doi.org/10.1109/IEMBS.2007.4352801

Kenfack Ngankam, H., Pigot, H., Lorrain, D., Viens, I., \& Giroux, S. (2020). Context awareness architecture for ambient-assisted living applications: Case study of nighttime wandering. Journal of Rehabilitation and Assistive Technologies Engineering, 7, 2055668319887864. https://doi.org/10.1177/2055668319887864

Konis, K., Mack, W. J., \& Schneider, E. L. (2018). Pilot study to examine the effects of indoor daylight exposure on depression and other neuropsychiatric symptoms in people living with dementia in long-term care communities. Clinical Interventions in Aging, 13, 1071-1077. https://doi.org/10.2147/CIA.S165224

Koumakis, L., Chatzaki, C., Kazantzaki, E., Maniadi, E., \& Tsiknakis, M. (2019). Dementia care frameworks and assistive technologies for their implementation: A review. IEEE Reviews in Biomedical Engineering, 12, 418. https://doi.org/10.1109/RBME.2019.2892614

Malkin, J. (1992). Hospital interior architecture: Creating healing environments for special patient populations: Creating healing environments for special patient populations. Van Nostrand Reinhold.

Manera, V., Chapoulie, E., Bourgeois, J., Guerchouche, R., David, R., Ondrej, J., Drettakis, G., \& Robert, P. (2016). A feasibility study with image-based rendered virtual reality in patients with mild cognitive impairment and dementia. PloS One, 11(3), e0151487. https://doi.org/10.1371/journal.pone.0151487

Marquardt, G., Bueter, K., \& Motzek, T. (2014). Impact of the design of the built environment on people with dementia: An evidence-based review. HERD, 8(1), 127-157. https://doi.org/10.1177/193758671400800111 
McCullough, M. (2005). Digital ground: Architecture, pervasive computing, and environmental knowing (1st paperback ed.). MIT Press.

Montana, J. I., Tuena, C., Serino, S., Cipresso, P., \& Riva, G. (2019). Neurorehabilitation of spatial memory using virtual environments: A systematic review. Journal of Clinical Medicine, 8(10). https://doi.org/10.3390/jcm8101516

Moyle, W., Jones, C., Dwan, T., \& Petrovich, T. (2018). Effectiveness of a virtual reality forest on people with dementia: A mixed methods pilot study. The Gerontologist, 58(3), 478-487. https://doi.org/10.1093/geront/gnw270

Mrakic-Sposta, S., Di Santo, S. G., Franchini, F., Arlati, S., Zangiacomi, A., Greci, L., Moretti, S., Jesuthasan, N., Marzorati, M., Rizzo, G., Sacco, M., \& Vezzoli, A. (2018). Effects of combined physical and cognitive virtual reality-based training on cognitive impairment and oxidative stress in mci patients: A pilot study. Frontiers in Aging Neuroscience, 10, 282. https://doi.org/10.3389/fnagi.2018.00282

National Institutes of Health, National Institute on Aging. (2017, October 10). What happens to the brain in alzheimer's disease? https://www.nia.nih.gov/health/what-happens-brain-alzheimers-disease

Nesbitt, K., \& Nalivaiko, E. (2020). Cybersickness. In N. Lee (Ed.), Encyclopedia of computer graphics and games (pp. 1-6). Springer International Publishing; Imprint: Springer. https://doi.org/10.1007/978-3-31908234-9_252-1

Optale, G., Urgesi, C., Busato, V., Marin, S., Piron, L., Priftis, K., Gamberini, L., Capodieci, S., \& Bordin, A. (2010). Controlling memory impairment in elderly adults using virtual reality memory training: A randomized controlled pilot study. Neurorehabilitation and Neural Repair, 24(4), 348-357. https://doi.org/10.1177/1545968309353328

Pengas, G., Williams, G. B., Acosta-Cabronero, J., Ash, T. W. J., Hong, Y. T., Izquierdo-Garcia, D., Fryer, T. D., Hodges, J. R., \& Nestor, P. J. (2012). The relationship of topographical memory performance to regional neurodegeneration in alzheimer's disease. Frontiers in Aging Neuroscience, 4, 17. https://doi.org/10.3389/fnagi.2012.00017

Pot-Kolder, R., Veling, W., Counotte, J., \& van der Gaag, M. (2018). Anxiety partially mediates cybersickness symptoms in immersive virtual reality environments. Cyberpsychology, Behavior and Social Networking, 21(3), 187-193. https://doi.org/10.1089/cyber.2017.0082

Sánchez, A., Millán-Calenti, J. C., Lorenzo-López, L., \& Maseda, A. (2013). Multisensory stimulation for people with dementia: A review of the literature. American Journal of Alzheimer's Disease and Other Dementias, 28(1), 7-14. https://doi.org/10.1177/1533317512466693

Smith, R. \& Watkins, N. (2016). Therapeutic environments. WBDG. https://www.wbdg.org/resources/therapeuticenvironments

Strong, J. (2020). Immersive virtual reality and persons with dementia: A literature review. Journal of Gerontological Social Work, 63(3), 209-226. https://doi.org/10.1080/01634372.2020.1733726

Topo, P. (2009). Dementia, design and technology: Time to get involved. (Assistive technology research series: vol. 24). IOS Press.

Torrington, J. M., \& Tregenza, P. R. (2007). Lighting for people with dementia. Lighting Research \& Technology, 39(1), 81-97. https://doi.org/10.1177/1365782806074484

van Hoof, J., Demiris, G., \& Wouters, E. J. (Eds.). (2017). Handbook of Smart Homes, Health Care and Well-Being. Springer International Publishing. https://doi.org/10.1007/978-3-319-01583-5

White, P. J. F., \& Moussavi, Z. (2016). Neurocognitive treatment for a patient with alzheimer's disease using a virtual reality navigational environment. Journal of Experimental Neuroscience, 10, 129-135. https://doi.org/10.4137/JEN.S40827

Yates, L., Csipke, E., Moniz-Cook, E., Leung, P., Walton, H., Charlesworth, G., Spector, A., Hogervorst, E., Mountain, G., \& Orrell, M. (2019). The development of the promoting independence in dementia (pride) intervention to enhance independence in dementia. Clinical Interventions in Aging, 14, 16151630. https://doi.org/10.2147/CIA.S214367 
Zeisel, J. (2000). Environmental design effects on alzheimer symptoms in long-term care residences. World Hospitals and Health Services: The Official Journal of the International Hospital Federation, 36(3), 27-31, 36, 38.

https://www.researchgate.net/publication/12056398_Environmental_design_effects_on_Alzheimer_sy mptoms_in_long-term_care_residences

Zeisel, J. (2003, July). Evidence-based design in coordinated health treatment. In Design \& Health World Congress \& Exhibition (WCDH 2003, Montreal). The International Academy for Design and Health. https://www.brikbase.org/sites/default/files/19John-Zeisel-WCDH-2003_0.pdf

\footnotetext{
${ }^{1}$ Brain plasticity is the ability of the brain to modify its connections or rewire itself. Without this ability, any brain, not just the human brain, would be unable to develop from infancy through to adulthood or to recover from brain injury.

${ }^{2}$ Exergames is a portmanteau of 'exercise' and 'gaming'), or gamercising, is a term used for video games that are also a form of exercise.
} 